\title{
A minicomputer system for multiperson computer-assisted telephone interviewing
}

\author{
GERALD H. SHURE and ROBERT J. MEEKER \\ University of California, Los Angeles, California 90024
}

\begin{abstract}
A computer-assisted telephone interviewing (CATI) system has been developed which has new and extensive implications for almost every phase of survey research. CATI provides (1) schedule design assistance, (2) new interviewing capabilities and controls which eliminate procedural errors, (3) coding assistance, (4) sampling administration, (5) interview supervision, and (6) postinterview data processing.
\end{abstract}

In little more than a decade, the telephone survey has become a major scientific tool for conducting survey research studies. It has been used in almost every conceivable variety of market, opinion, and attitude study on public issues and private concerns including education, health, crime, race relations, family planning, etc. Few issues, including highly sensitive social and personal ones, have escaped study by this method.

A major factor behind the growing, widespread use of phone interviewing has been the cost advantages in comparison with personal interviewing. However, along with the advantages claimed for telephone interviewing, increased concern over the quality of information obtained compared to that obtained in face-to-face interviewing has been voiced. One of the major concerns has been the adequacy of control over interview procedures and interviewer-respondent interactions.

The computer-assisted telephone interviewing (CATI) system described in this paper is designed to apply advanced interactive computing technology to the solution of the control problem and to the extension of research capabilities in telephone survey interviewing so as to broaden the system's scope and quality and further reduce cost.

The CATI system incorporates a comprehensive set of refinements and capabilities to support telephone interviewing. Figure 1, a schematic diagram of the system, indicates how the various programs interface with one another. These programs are discussed in terms of the major functions accomplished at three stages of

Development of a prototype of the CATI system was supported by a grant from the Russell Sage Foundation. The development of the current system is being supported by Grant SOC77-00190 from the National Science Foundation to the first author. This grant is one of several concerned with the general development and improvement of survey research methodology that is under the direction of Dr. Murray Aborn at the National Science Foundation. Appreciation is expressed to Dr. Miles S. Rogers and Craig M. Rogers for their creative programming contributions in developing CATI, and to Professor Donald J. Treiman and Douglas Longshore for their helpful comments on the manuscript. the interviewing activity: (1) the preinterview schedule design stage, (2) the interviewing proper stage, and (3) the postinterview data processing stage. These interlocking programs, all written in FORTRAN IV, operate on a PDP-11/45 computer under the RSX-11D and RSX-11M operating systems.

\section{PREINTERVIEW QUESTIONNAIRE SCHEDULE DESIGN STAGE}

The first of the functional areas is composed of two programs designed to assist investigators in preparing schedules for computer administration. The system neither assumes nor requires any knowledge of programming, but the user should be an expert in designing survey schedules.

The schedule editor and interview program generator programs are used to convert a written interview schedule into an interview sequence appearing on the computer console screen at the command of the interviewer. The schedule editor transforms a written interview schedule into an interview specification file; the interview program generator then uses this file to create the specific interviewing program which governs the display of items and the recording of responses during the interview itself.

The first step facing the investigator is that of producing the interview specification file. These specifications must be in a form that will be machine-readable by the interview program generator. At the same time, it is very important to make this specification process virtually the same as that used in designing fieldadministered interview schedules. Ease of specification is one of the important features of the system.

The content of the interview schedule prepared for entry into the computer is essentially the same as that found in a printed survey schedule. Only two modifications are required: (1) The answer codes for respondent choices must be placed in angle brackets, and (2) each question must terminate with a response arrow which is formed by two or more equal signs and a right-angle bracket, as indicated in the example below. 


\section{ARE YOU CURRENTLY EMPLOYED?$$
\langle 1\rangle \text {.- Yes }
$$$$
\langle 2\rangle \text {.. No }
$$$$
=\Rightarrow
$$

Aside from these, no other modification of content is required. Procedural instructions normally directed to the interviewer but performed by the computer are written in a form similar to that used in printed sched. ules and are placed in square brackets directly associated, as appropriate, with a given response. The example below tells the program to branch to Item $6 \mathrm{~A}$ if the answer is YES.

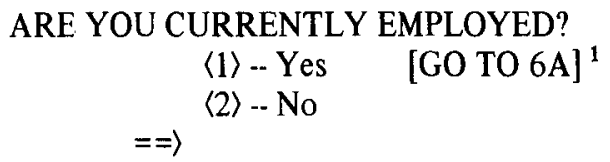

When we say that these procedures do not require programming, we mean the system provides a bridge between the usual conventions of preparing interview schedules and FORTRAN IV compilable code. The bridge is an automatic translator in the interview program generator. It takes in an interview schedule specification file and puts out FORTRAN code (the interviewing program) that, when compiled and executed,

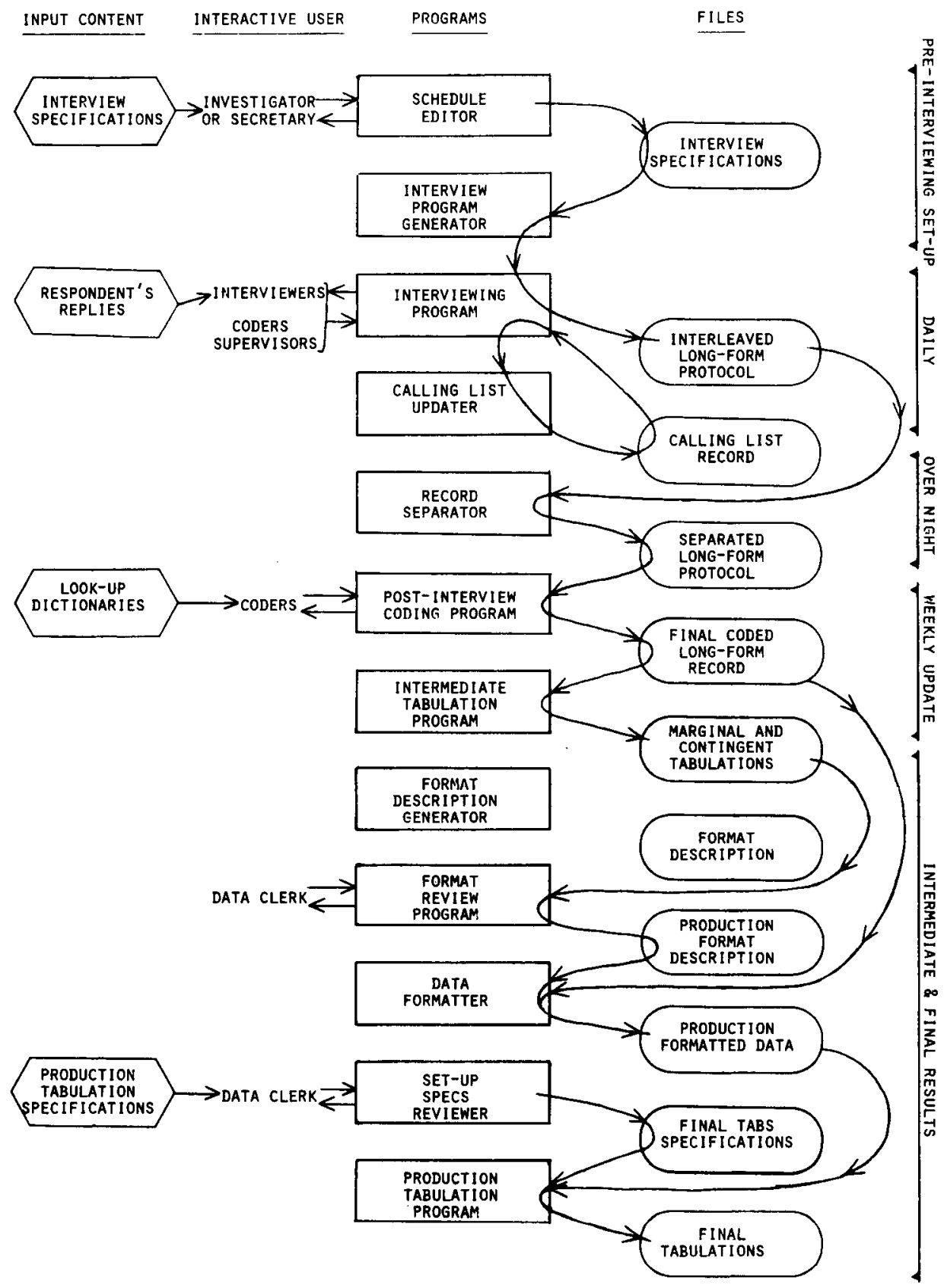

Figure 1. Schematic of proposed system for computer-assisted telephone interviews. 
implements the schedule through the computer. The translator in the prototype interview program generator is first generation; though it successfully recognizes most item forms and procedures found in interview schedules, new forms undoubtedly will emerge. The program can be easily extended to incorporate these. Furthermore, as the new capabilities planned for the interviewing program are added, conventions must be specified and the interviewer program generator modified to recognize and process these to provide the desired capabilities. Of course, it will never be completely comprehensive in its ability to translate interview schedules. For that reason, there are advantages in having FORTRAN code as an intermediate step since that code, with relatively little programming effort, can be modified to deal with idiosyncratic applications. Modular construction of the system also provides for on-site addition of modules programmed by investigators who wish to add new capabilities. The system, at present, relies on the availability of a general-purpose editor for creating and modifying the schedule specifications file on-line, or the file may be entered on cards or tape. A special-purpose editor that includes functions specific to schedule file editing is planned for the near future. The preinterviewing system follows the design strategy of a more general-purpose system for implementing on-line experiments (Shure \& Meeker, 1974).

\section{INTERACTIVE INTERVIEWING STAGE}

The second major functional component of the system provides computer assistance during the interviewing proper phase and currently supports up to 15 simultaneous positions manned by phone interviewers. Interviewers sit before a computer terminal on which questions, probes, lists, and interview instructions are presented as displays. After reading the question to the respondent, the interviewer uses the terminal to record the obtained response. This system operates in a multistation mode supporting 15 console positions. The positions may be occupied by supervisors, monitors, data entry clerks, and coders, as well as by interviewers who are served concurrently by the same program. The positions can also be allocated to run more than one survey at the same time.

There are three principal advantages of the multistation mode over a mode in which each is served by a separate version of the program. First, it is more efficient in terms of using computer resources; the more copies of the program needed for simultaneous usage, the more computer memory required. Second, the shared mode does not require a timeshared computer; the multicopy mode does. And third, with all interviewers served by one program, it is much easier to incorporate supervisory, administrative, research, and other functions that require easy communication between interviewers and supervisors or among concurrent users. When each interviewer is operating independently under separate program control, it is more difficult to effect interaction between the supervisor and interviewers that will not be functionally disruptive. With all users under single program control, it is much easier to insure that the occasions and timing of interactions are as unobtrusive as possible. The multistation mode has much to recommend it, but perhaps the most important consideration is that the generated FORTRAN code, in effect, performs as a special-purpose mini timesharing system that will execute on a nontimeshared machine. As a result, it is transferable to either a timeshared or dedicated computer system.

Any special utilities required for execution have been isolated wherever possible; output procedures that might be dependent on equipment configurations in a given installation have also been isolated for easing adaptation to other sites. At the present time, multistation versions of the system have been adapted for the Census Bureau and the University of California Survey Research Center at Berkeley.

Within this general framework, the interactive interviewing CATI provides efficiencies and capabilities significantly beyond those achieved in telephone survey interviewing. These include:

Improved control over standard interview processes through basic capabilities for specifying a variety of item presentations and formats, response coding, error checking, and contingency branching based on prior response patterns. These capabilities eliminate most interviewer errors associated with skipping of items and recording of illegal or inconsistent responses. As a consequence, they virtually eliminate the major sources of error requiring the costly postinterview error checking and the even more costly procedures involved in postinterview attempts to obtain correct or missing information.

Consistency checks over the course of the interview to detect discrepancies in respondent replies to different items. The consistency check procedures can also be used to determine the presence of response sets based on the occurrence of defined patterns over a set of items. On detection of "suspect" patterns of response, these can be readministered automatically at subsequent points in the interview in similar or altered formats to check validity. More generally, when errors or inconsistencies are revealed, provisions are available for the interviewer to back up to earlier questions, to review, and, if necessary, to correct earlier responses.

Provisions for interviewer to intersperse comments at any juncture as a means of interjecting an observation for the record and to permit the computer to associate these comments with particular responses to items for purposes of selective retrieval. This capability not only provides a simple means for registering important qualifying comments, but it also provides for their systematic elicitation at appropriate stages in postinterview data processing.

Abilities to systematically vary the order of presenta- 
tion of items across interviews and to assess and control order effects. This capability illustrates rather well the potential of CATI for control and research. The effect of questionnaire item order on response has been discussed for more than two decades in both the survey and psychological testing literature. The potential for bias due to item order may exist for a number of reasons. Earlier items may affect later ones because of primacy or recency learning effects, acquiescence sets or opposition response sets, or triggering of commitments that lead to subsequent response distortion due to maintenance of a previously stated position. In another vein, the effects of placements of particular items in the beginning, middle, or last third of the questionnaire have evoked concern over potential associated effects of boredom or fatigue. This concern is particularly relevant because of the increased use of large omnibus surveys.

In recognition of these potential sources of order of item biases, survey interview manuals emphasize the importance of maintaining strict adherence to the prescribed question order from interview to interview. When followed, this rule at least helps to standardize the effects of item order for all respondents to the item order in the particular schedule; it does not, however, minimize or detect the presence of such bias. It is discomforting to realize how little has actually been done to assess the impact of item order or to counterbalance the effects of such bias by systematic variation in item presentation order.

The reason for this state of affairs is obvious. The numbers of different sequences of $n$ items is $n$ !. For anything beyond three items, presentation of all counterbalanced sequences requires producing a sizeable number of different forms of the printed schedules for each unique sequence or requires using procedural branching rules for administering the appropriate order variant. Both of these procedures are burdensome, costly, and significantly increase the likelihood of errors in administration, recording of replies, and subsequent data processing.

With computer-administration, random or systematically counterbalanced variations in item presentation order can be achieved for small or large subsets of items, for blocks of items, and for combinations of both of these. In execution, the program not only presents the items in the appropriately varied order, but also records the particular order variant and stores the results with proper (explicit or implicit) item identification and order position. The implementation of this form of variable ordering of list presentation allows control and evaluation of order effects on a scale beyond anything previously considered or attempted.

Assistance in presentation, recording, and coding of different forms of open-ended items. Open-nded questions have been used, albeit reluctantly, in survey research where precoding of replies cannot be determined beforehand (i.e., where the set is not yet exhaus- tively defined) or where precoding involves too large and/or too complex a set of responses. But even where precoded responses are used, the interviewer often is instructed to elicit a codable answer by additional questioning or probing when a respondent's initial answer does not match one of the preselected codes for a question. Presently, with little effective standardization in the use of probes by interviewers, the information obtained for items such as these is subject to interviewer bias and frequently lacks the specificity or precision required for subsequent coding, particularly where items are open ended. Because of concern over the quality of interviewer coding skills and over potential interference with interview rapport that coding-associated delays might produce, coding itself is rarely attempted during the interview.

Improved and systematic procedures for elicitation of information for coding open-ended responses and procedures to routinize simple code assignments are areas in which computer support would be highly useful. The kind of coding support that the computer would provide varies with the properties of the openended question and with the extent to which amplifying information may be usefully collected during the interview. On this basis, four classes of open-ended items have been distinguished. Three of the coding schemes described below are planned for development as part of the proposed project; the fourth is noted as a candidate for future development within the system.

1. Coding responses into growing lists. This simplest form of computer-assisted coding is a direct benefit of the fact that all responses are being recorded directly in machine-readable form. Coding merely involves automatic assignment of numerical item codes to each unique element in the total set of responses. Codes need not be specified in advance. Presumably, this type of coding would be done by the computer after each interview session. The coding need not be deferred until all unique responses have been gathered since the unique elements list and associated codes are retained and updated from one session to the next. As each new response is checked, the computer determines if it is already on the list; if so, the coded value for the respondent is entered. If the new entry is not on the list, then a new code is added to the list by the computer, and the new code is also automatically entered for the respondent. At this juncture, all new codes may be reviewed for multiple codes erroneously assigned to the same element due to spelling variations or typing errors, and corrections may be made. This coding procedure is primarily viewed as a means of sharply reducing errors and effort associated with straightforward "look up" and assignment of codes.

2. Coding responses into categories that are defined and modified by new instances. At the next level of computer-assisted coding, the automatically derived codes for each unique element are further encoded or 
directly encoded into a set of categories. Examples of such lists might be those for categories of illnesses, professional organizations, magazines, etc. What the investigator wants is a categorization of the replies rather than mere enumeration, as produced with the growing list procedure. In processing each element, it would be compared with previously classified elements: If a match was found it would be automatically classified; if it was not so matched, a classification would be elicited from a coder interacting with the program, leading to classification under an existing category or, more rarely, creation of a new one. Presumably this processing becomes more and more automatic as the study proceeds, until only idiosyncratic cases require any coder review.

3. Coding responses using information systematically elicited from the respondent. This class of coding assistance aims to elicit specific additional information from the respondent to help resolve ambiguities or doubts in coding-a task investigators have been loathe to undertake because the administration and control of probes have proved too unwieldy and unreliable. (It differs from the two preceding approaches, where the computer merely provides clerical assistance by automating "look up" and assignment of codes or where selective use of knowledge of the coder is used to improve the efficiency and completeness of computer coding.) The approach presupposes that the investigator, through prior analysis of the subject matter domain, can specify the critical dimensions of the encoding to permit the computer to lead the interviewer through successive questions and choices until a final encoding can be made. Coding thus becomes a part of the interview process with highly structured branching specifications.

An example of a domain where the proposed approach is applicable is that of industrial and occupational coding into U.S. Census categories. There are over 200 industrial and 400 occupational titles that are mutually exclusive and exhaustive. Since these titles do not conform to a simple classification scheme, a complex, branching logic is required to determine the current code. This general area of application is one in which computer assistance would yield a great advantage by permitting the coding to be done in direct interaction with the respondent to narrow down the options and arrive at a final category assignment.

4. Postinterview coding of responses for incompletely defined categories. The proposed coding solutions for the three preceding classes of open-ended items may be of little value for some open-ended questions that cannot be circumscribed in advance and that do not have a final set of coding categories decided on. However, postinterview manual coding such as is traditionally employed is extremely time-consuming and costly and frequently requires sophisticated coding skills. Furthermore, when the coding task is well underway or completed, it is frequently determined that revised or totally different categorization schemes would be more appropriate or of additional value. As a consequence, investigators are loathe to use such items even though they can be of immense value in exploratory aspects of a study.

Frisbie and Sudman (1968) first proposed the use of computer-assisted content analysis for performing the coding of such items. A major barrier to such use is the costly and time-consuming procedure involved in getting the data in to the computer. Not only must one go back to each of the questionnaires to find the page on which the answer is written, but these must be keypunched or prepared in some manner for computer processing.

The computer-assisted telephone interviewing system eliminates this major hurdle, since all open-ended responses will exist in machine-readable form at the close of the interview. The elimination of this barrier may do much to stimulate the use of an important, but rarely used, inductive tool to analyze open-ended replies. ${ }^{2}$

Use of more complex branching processes and algorithms to improve interview procedures. More efficient and precise algorithms may be introduced for eliciting information from the respondent and for controlling the interview process that otherwise would be too complex, procedurally cumbersome, or time consuming for the interviewer to effect without computer assistance. Many refinements derive from increased opportunity for sophisticated "branching" within the interview. Branching occurs when an answer to one or more earlier questions determines the sequence of later questions. This procedure, though often desirable from a data collection standpoint, unfortunately can be a common source of interviewer error and confusion. Rather than risk such confusion, interview designers frequently retain procedures that involve unnecessary data collection in order to achieve a simple, slavish uniformity.

For similar reasons, excessive computational demand on the interviewer is also avoided and, as a consequence, may lead to the use of procedures based on approximations rather than those based on more exact algorithms. An example of this is seen in the preprogrammed "selection table" for choosing a respondent within a household (Bryant, 1975). For example, use of Bryant's procedures eliminates the possibility of ever selecting a woman as a respondent who is not the oldest or youngest in a household with three or more women.

If the interview is computer assisted, it is a simple matter to provide the combination of branching and calculation to implement the precise algorithm, and to do so with even less procedural difficulty than that encountered in using less adequate approximation procedures now employed in many studies.

Calling list updater for administration of sampling and respondent selection procedures including random digit dialing $(R D D)$. This program governs the management of sampled telephone numbers, permitting on-line RDD sampling or assignment from lists generated off- 
line. The program automatically assigns numbers for calling within any specified range of digits with given telephone area codes or exchanges, keeps track of numbers requiring call-back reassignment, records the disposition of calls, etc.

In addition to the savings realized by locating and contacting respondents by telephone instead of by field visits that require travel time and transportation costs, the use of RDD as a means of drawing a sample (Tuchfarber \& Klecka, 1976) is not only much less costly than the drawing of a sample for a field survey, but it is also a less complex procedure than that used in obtaining a representative household sample. RDD virtually eliminates listing errors that occur in the preparation of a sampling frame and affords greater control over the interviewer's role in household selection (Eastlack \& Assael, 1966).

Procedures for improved centralized supervision and administration of interviewing. A major increase in the potential for supervision takes place with the incorporation of the supervisory station into the multistation system. As an integral part of the system, the supervisor, at his or her computer console, is able to monitor the step-by-step progress of each interview, to view the computer presentations currently displayed to any selected interviewer, and to monitor all interviewer behavior. As desired, the computer can automatically alert the supervisor to the upcoming occurrence of specific items to be monitored more closely by TV or audio means. This provision adds greatly to the efficiency of phone "tap-in" monitoring.

Interviewing timing data. Timing information on responses to individual items, for example, response latency or amount of time needed to reply, is rarely collected in face-to-face interviews. The decision not to do so is probably based on the concern that it might interfere with interview rapport and because of the additional burden it imposes on the interviewer. There is also a question of the reliability of timing data obtained under these conditions.

Within the framework of the computer-assisted interview, the aforementioned liabilities are eliminated and such data are easily and reliably collected. The precise value of this information in survey research has yet to be assessed, but such data could be of immense value for some aspects of survey interviewing, particularly where multiple, method-divergent measures are rare. For instance, with an adequate reference base on item response time, modal timing data for particular items might be studied together with answers to gauge interviewee resistance, carelessness, or lying. The effect of item formats, procedures, and interview styles might also be examined with these data. For example, consistent patterns of variability among interviewers in interview duration or in response time with particular kinds of items may prove useful in pinning down important sources of response variability. The phenomenon of procedural speed-up that may occur when an interviewer repeatedly administers the same schedule, an occurrence noted where there is extensive repetition of instructions in a psychological experiment, could also be examined critically. Indeed, the variety of research purposes that timing data may possibly serve will only fully emerge after some experience with the collection of these data and the design of special items and procedures that takes full advantage of timing information.

\section{POSTINTERVIEW PROCESSING PROGRAMS}

The third functional component of CATI is a collection of linked programs for on-line data processing, including cleaning, coding of open-ended responses, and formatting and labeling of the variables so that the data can be input directly into standard statistical programs. As these programs represent data processing functions more familiar to the reader, the subprograms shown in Figure 1 will not be reviewed in any detail at this time. All of these tasks represent a significant portion of survey costs that are not presently being reduced by electronic data processing because the information is not directly accessible in machine-readable form. Furthermore, they introduce an additional capabilitythe dynamic evaluation and pooling of data. Interviewers on-line in the same system contribute to a common data base that is immediately accessible within the system. The on-line data pool permits relatively frequent analysis and sensing for important respondent characteristics (special study cases, significant outliers, etc.) for immediate augmentation of the interview or flagging for follow-up, which significantly reduces the cost of many special sample studies. The data base in constantly updated form can also help to avoid oversampling in the concluding stages of a study by allowing interviewing to be restricted to relevant cases only.

\section{SUMMARY}

This paper describes a computer-assisted interviewing system for telephone survey research. It describes some of the practical and research implications of the new and powerful extension of interviewing capabilities and procedural controls which promise to significantly enhance the present state of the art for dealing with problems of reliability, validity, cost, and scope of telephone interviewing. Of equal importance to the survey interviewing capabilities provided by CATI is the unique manner in which CATI opens up survey interviewing procedures to more critical examination by experimental research. The centralized location of the interviewing staff, combined with on-line computer monitoring and controlled presentation of items, allows each facet of the interviewing process to be scrutinized while the interview is being conducted and thus presents 
the opportunity to pursue a more fundamental understanding of the method itself.

\section{REFERENCES}

BryaNT, B. E. Respondent selection in a time of changing household composition. Journal of Marketing Research, 1975, 12, 129-135.

Eastlack, J. O., JR., \& Assael, H. Better telephone surveys through centralized interviewing. Journal of Advertising Research, 1966, 6, 2-7.

Frisbie, B., \& Sudman, S. The use of computers ' in coding free responses. Political Opinion Quarterly, 1968, 32, 216-232.

Rogers, C. M., Rogers, M. S., Meeker, R. J., Greenstein, S., \& MineR, R. L. TERS-Text encoding and retrieval system. Data: Its use, organization and management. Proceedings of the 1975 Pacific Regional Conference of the ACM, 1975, 50-55.

ShURE, G. H., \& MeEkER, R. J. Laboratory experimentation at the Center for Computer-Based Behavioral Studies. Behavior Research Methods \& Instrumentation, 1974, 6, 241-248.

Tripodes, P. G., Greenstein, S., Dolan, P., Bodnaras, M., \& SHure, G. H. Automatic content coding of English text. Proceedings of the 1974 Annual Conference of the Association for Computer Machinery, 1974, 426-434.
Tuchfarber, A. J., JR., \& KLECKA, W. R. Measuring crime victimization: An efficient method. Washington, D.C: The Police Foundation, 1976.

\section{NOTES}

1. All of the skipping instructions that appear in a manually administered schedule and their counterparts in the computer-specification version are, of course, absent in the computer-administered version used by the interviewer, who sees only the results of the branching processes and need not be concerned with their implementation.

2 . In this regard, a newly completed text processing system (Rogers, Rogers, Meeker, Greenstein, \& Miner, 1975) developed at the Center for Computer-Based Behavioral Studies should further stimulate the use of computer-assisted content analysis. The system runs on the same PDP-11 computer used for the development of the telephone interviewing system. The text analysis system not only provides content analysis capabilities, but it also provides interactive text editing and retrieval capabilities, thus greatly reducing the time and effort required to clean and prepare data for the particular content analysis desired. Another component of the system attempts to overcome the inherent weakness of traditional term-byterm content analysis by also operating on thematic (syntactic) units within a sentence (Tripodes, Greenstein, Dolan, Bodnaras, \& Shure, 1974). 